\begin{tabular}{|c|l|}
\hline Title & Noninvertible Bogoliubov transformations and instability of embedded eigenvalues \\
\hline Author(s) & A rai, A sao \\
\hline Citation & Journal of Mathematical Physics, 32(7), 1838-1846 \\
\hline https:/doi.org/10.1063/1.529248 \\
\hline Issue Date & 1991-07 \\
\hline Doc URL & http://hdl.handle.net/2115/13675 \\
\hline Rights & Copyright $\odot$ 1991 American Institute of Physics \\
\hline Type & article \\
\hline File Information & jmp32-7.pdf \\
\hline
\end{tabular}

Instructions for use 


\title{
Noninvertible Bogoliubov transformations and instability of embedded eigenvalues
}

\author{
Asao Arai \\ Department of Mathematics, Hokkaido University, Sapporo 060. Japan
}

(Received 28 August 1990; accepted for publication 23 January 1991)

\begin{abstract}
A class of noninvertible Bogoliubov transformations in an abstract Boson Fock space is used to construct in the Fock space a family of self-adjoint operators $H$ which are quadratic in the annihilation and the creation operators and are of the form $H=H_{0}+H_{1}$ with the property that the unperturbed part $H_{0}$ may have embedded-eigenvalues unstable under the perturbation $H_{l}$. Scattering theory associated with the pair $\left(H_{0}, H\right)$ is also discussed. In application to quantum field theory, the family of the operators $H$ gives a unified description for the Hamiltonians of models of a quantum harmonic oscillator coupled to a quantized scalar or radiation field.
\end{abstract}

\section{INTRODUCTION}

A Bogoliubov transformation in a Fock space is a transformation of the annihilation and the creation operators which is expressed linearly in terms of them and preserves the (anti-) canonical commutation relations (CCR). There have been a number of studies on proper Bogoliubov transformations so far (e.g., Refs. 1-3 and references therein). In this paper we are concerned with another type of Bogoliubov transformations that may be called noninvertible Bogoliubov transformations: A Bogoliubov transformation is said to be noninvertible if there exist no invertible bounded linear operators that implement it on the Fock space under consideration. It seems that attention has not been paid so much to noninvertible Bogoliubov transformations or at least they have not been fully exploited. In the present paper we consider a class of noninvertible Bogoliubov transformations in connection with a type of singular perturbation of self-adjoint operators acting in Fock space (see below). The class of Bogoliubov transformations under study is defined in the Boson (symmetric) Fock space $\mathscr{F}_{s}(\mathscr{H})$ over $\mathscr{H}=\mathscr{K} \oplus \mathscr{M}$ (the direct sum of two Hilbert spaces $\mathscr{K}$ and $\mathscr{M})$, which is identified with $\mathscr{F}_{s}(\mathscr{K}) \otimes \mathscr{F}_{s}(\mathscr{M})$. We shall show that the noninvertible Bogoliubov transformations can be used to construct in $\mathscr{F}_{s}\left(\mathscr{F}^{S}\right)$ a family of self-adjoint operators $H$ which are quadratic in the annihilation and the creation operators and are of the form $H=H_{0}+H_{I}$ with the following properties.

(i) The "unperturbed" part $H_{0}$ is of the form

$H_{0}=d \Gamma(h) \otimes I+I \otimes d \Gamma(l)$,

where $h$ and $l$ are non-negative self-adjoint operators in $\mathscr{K}$ and $\mathscr{M}$, respectively, and $d \Gamma(A)$ (resp. $I$ ) denotes the second quantization of $A$ (resp. identity).

(ii) For a real constant $E, H-E$ is unitarily equivalent to $d \Gamma(h)$ acting in $\mathscr{F}(\mathscr{K})$.

To see what the above result implies, consider, e.g., the case where $\sigma(h)$, the spectrum of $h$, is purely continuous with $\sigma(h)=\left[\omega_{0}, \infty\right)\left(\omega_{0} \geqslant 0\right.$ : a constant $)$ and $\sigma(l)$ is purely discrete. Then we have

$$
\begin{aligned}
& o(d \Gamma(h))=\{0\} \cup\left[\omega_{0}, \infty\right), \quad \sigma_{p}(d \Gamma(h))=\{0\}, \\
& \sigma(d \Gamma(l))=\sigma_{p}(d \Gamma(l))=\left\{E_{n}\right\}_{n=0}^{\infty},
\end{aligned}
$$

with $E_{n} \geqslant 0\left(E_{0}=0\right)$, where $\sigma_{p}(\cdot)$ denotes point spectrum.
Hence,

$$
\begin{aligned}
& \sigma\left(H_{0}\right)=\left\{E_{n}\right\}_{n=0}^{\infty} \cup\left[\omega_{0}, \infty\right), \\
& \sigma_{p}\left(H_{0}\right)=\left\{E_{n}\right\}_{n-0}^{\infty},
\end{aligned}
$$

which mean that each $E_{n}$ is also an eigenvalue of $H_{0}$ and the eigenvalues $E_{n} \geqslant \omega_{0}$ are embedded in the continuous spectrum of $H_{0}$ (we call such eigenvalues embedded eigenvalues). On the other hand, (ii) implies that

$$
\sigma(H)=\{E\} \cup\left[E+\omega_{0}, \infty\right), \quad \sigma_{p}(H)=\{E\} .
$$

Thus all the embedded eigenvalues $E_{n} \geqslant \omega_{0}$ turn out to disappear under the perturbation $H_{l}$, i.e., they are unstable under the perturbation (we may regard $E_{n}<\omega_{0}$ as eigenvalues changing to $E$ under the perturbation $H_{I}$ ). In this sense the perturbation $H_{f}$ is singular relative to $H_{0}$. In this way each of the noninvertible Bogoliubov transformations under consideration can have a connection with instability, under a perturbation, of embedded eigenvalues of a self-adjoint operator in the Fock space $\mathscr{F}_{5}(\mathscr{H})$. The present abstract theory has application to quantum field theory (QFT). In fact, in concrete realizations of $\{\mathscr{F}, \mathscr{H}\}$, the class of $H$ gives a unified description for the Hamiltonians of models of a quantum harmonic oscillator coupled to a quantized scalar or radiation field, ${ }^{4-8}$ where the unperturbed part of each of those Hamiltonians is of the form (1.1). The abstract theory developed in this paper clarifies the mathematical structure of those models, giving us a satisfactory understanding of them.

We should mention that the idea of the present work is already implicit in a previous paper (Ref. 8) where an abstract and unified formulation is given for models of a onedimensional quantum harmonic oscillator coupled to a quantized scalar field. The present paper gives, with a generality, an extension and a refinement of results in Ref. 8. In applications of the present formulation to models mentioned above, the harmonic oscillator is not necessarily one dimensional.

The outline of the present paper is as follows. Section II is a preliminary section and is of review nature. We define basic objects in an abstract Boson Fock space and summarizes some fundamental facts. After introducing in Sec. III the class of noninvertible Bogoliubov transformations to be considered and discussing some of their properties, we con- 
struct in Sec. IV a family of self-adjoint operators $H$ with the properties described above. Section V is devoted to scattering theory associated with the pair $\left(H_{0}, H\right)$. In the last section we mention some examples in QFT.

Some general symbols used in the present paper are (', *): inner product (linear in $*$ ); $\|\cdot\|:$ norm of Hilbert space; $\|A\|$ : operator norm of the operator $A ; D(A)$ : domain of the operator $A ; \boldsymbol{B}\left(\mathscr{H}_{1}, \mathscr{H}_{2}\right)$ : the space of all bounded linear operators from a Hilbert space $\mathscr{H}_{1}$ to a Hilbert space $\mathscr{H}_{2}$; and $\mathbf{B}(\mathscr{H}):=\mathbf{B}(\mathscr{H}, \mathscr{H})$.

\section{FUNDAMENTAL FACTS IN AN ABSTRACT BOSON FOCK SPACE}

We first recall the definition of some objects in an abstract Boson Fock space (e.g., Refs.1; Ref. 9, Sec. II.4; Ref. 10, Sec. X.7). Let $\mathscr{H}$ be a separable complex Hilbert space and $S^{n}(\mathscr{H})=\otimes_{s}^{n} \mathscr{H}$ be the $n$-fold symmetric tensor product of $\mathscr{H}$ with $S^{0}(\mathscr{H})=\mathbb{C}$. The Boson (symmetric) Fock space $\mathscr{F}_{s}(\mathscr{H})$ over $\mathscr{H}$ is defined by the completed infinite direct sum of $S^{n}(\mathscr{H})$ :

$$
F_{s}(\mathscr{H})=\oplus_{n=0}^{\infty} S^{n}(\mathscr{H}) \text {. }
$$

We denote by $A(F), F \in \mathscr{H}$, the annihilation operator in $\mathscr{F}_{s}(\mathscr{H})$ (antilinear in $\left.F\right)$. Let $\Omega=\{1,0,0, \ldots,\} \in \mathscr{F}_{s}(\mathscr{H})$ be the Fock vacuum and let

$$
\begin{aligned}
\mathscr{F}_{\mathrm{fin}}(\mathscr{H})= & \mathscr{L}\left\{A\left(F_{1}\right)^{*} A\left(F_{2}\right)^{*} \cdots A\left(F_{n}\right) * \Omega,\right. \\
& \left.\Omega \mid F_{j} \in \mathscr{H}^{*}, j=1, \ldots, n, n \geqslant 1\right\},
\end{aligned}
$$

where $\mathscr{L}\{\cdots\}$ denotes the subspace algebraically spanned by vectors in $\{\cdots\}$. We denote by $A(F)$ either $A(F)$ or $A(F)^{*}$. The operators $A(F)^{\#}$ leave $\mathscr{F}_{\text {fin }}(\mathscr{H})$ invariant satisfying the $\mathrm{CCR}$

$$
\left[A(F), A(G)^{*}\right]=(F, G), \quad[A(F), A(G)]=0, \quad F, G \in \mathscr{H},
$$

where $[A, B] \equiv A B-B A$.

We next define operators quadratic in $A^{\#}$. Let $J$ be a conjugation on $\mathscr{H}$, i.e., $J$ is an antilinear isometry on $\mathscr{H}$ with $J^{2}=1$. For $F \in \mathscr{H}$ and $T \in \mathbb{B}(\mathscr{H})$, we define $\bar{F} \in \mathscr{H}$ and $\bar{T} \in \mathbb{B}(\mathscr{F})$ by

$$
\begin{aligned}
& \bar{F}=J F, \\
& \bar{T}=J T J .
\end{aligned}
$$

We denote by $\mathscr{F}_{2}\left(\mathscr{H}_{1}, \mathscr{H}_{2}\right)$ the space of HilbertSchmidt operators from a Hilbert space $\mathscr{H}_{1}$ to a Hilbert space $\mathscr{H}_{2}$. We set $\mathscr{I}_{2}(\mathscr{H})=\mathscr{I}_{2}(\mathscr{H}, \mathscr{H})$. For every $K \in \mathscr{F}_{2}(\mathscr{H})$, there exist (not necessarily complete) orthonormal sets $\left\{\psi_{n}\right\}_{n=1}^{M}$ and $\left\{\phi_{n}\right\}_{n=1}^{M}$ in $\mathscr{H}$ ( $M$ may be finite or infinite) and positive real numbers $\left\{\lambda_{n}\right\}_{n=1}^{M}$ such that

$$
\begin{aligned}
& \sum_{n=1}^{M} \lambda_{n}^{2}<\infty, \\
& K=\sum_{n=1}^{M} \lambda_{n}\left(\psi_{n}, \cdot\right) \phi_{n},
\end{aligned}
$$

where, in the case $M=\infty$, the sum in (2.6) converges in operator norm (e.g., Ref. 9, Theorems VI.17 and VI.22). We then define for finite positive integers $N$

$$
\left\langle A^{*}\left|K_{N}\right| A^{*}\right\rangle=\sum_{n=1}^{N} \lambda_{n} A\left(\bar{\psi}_{n}\right)^{*} A\left(\phi_{n}\right)^{*}
$$

and

$$
\left\langle A\left|K_{N}\right| A\right\rangle=\sum_{n=1}^{N} \lambda_{n} A\left(\psi_{n}\right) A\left(\bar{\phi}_{n}\right) .
$$

The following lemma is easily proved.

Lemma 2.1: For all $\Psi \in \mathscr{F}_{\text {fin }}(\mathscr{H})$, the strong limits

$$
s-\lim _{N \rightarrow \infty}\left\langle A^{*}\left|K_{N}\right| A^{*}\right\rangle \Psi \equiv\left\langle A^{*}|K| A^{*}\right\rangle \Psi
$$

and

$$
s-\lim _{N \rightarrow \infty}\left\langle A\left|K_{N}\right| A\right\rangle \Psi \equiv\langle A|K| A\rangle \Psi
$$

exist. Moreover, the operator $\left\langle A^{\#}|K| A^{\#}\right\rangle$ defined on $\mathscr{F}_{\text {fin }}(\mathscr{H})$ is closable and

$$
\left\langle A^{*}|K| A^{*}\right\rangle^{*}=\left\langle A\left|K^{*}\right| A\right\rangle \text { on } \mathscr{F}_{\text {fin }}(\mathscr{H}) \text {. }
$$

We denote the closure of $\left\langle A^{\#}|K| A^{\#}\right\rangle$ by the same symbol.

It easily follows from the definition of $\left\langle A \#|K| A^{\#}\right\rangle$ that

$$
\left\langle A^{*}|K| A^{\#}\right\rangle=\left\langle A^{\#}\left|\bar{K}^{*}\right| A^{\#}\right\rangle .
$$

Lemma 2.2: Let $K_{1}, \ldots, K_{n} \in \mathscr{F}_{2}(\mathscr{H})$. Then, for all $\Psi \in \mathscr{F}_{\text {in }}(\mathscr{H}), n \geqslant 1$, and for $F_{j} \in \mathscr{H}, j=1, \ldots, k, k \geqslant 1$,

$$
\Psi \in D\left(\left\langle A^{\#}\left|K_{n}\right| A^{\#}\right\rangle \cdots\left\langle A^{\#}\left|K_{1}\right| A^{\#}\right\rangle\right)
$$

and

$$
\left\langle A^{\#}\left|K_{n}\right| A^{\#}\right\rangle \cdots\left\langle A^{\#}\left|K_{1}\right| A^{\#}\right\rangle \Psi \in D\left(A\left(F_{1}\right)^{\# \cdots A\left(F_{k}\right)}\right) \text {. }
$$

Moreover, for all $K \in \mathscr{I}_{2}(\mathscr{H})$ and $F \in \mathscr{H}$,

$$
\begin{aligned}
& {\left[A(F)^{*},\left\langle A^{*}|K| A^{*}\right\rangle\right]=0,} \\
& {\left[A(F),\left\langle A^{*}|K| A^{*}\right\rangle\right]=A\left(K_{s} \bar{F}\right)^{*}}
\end{aligned}
$$

on $\mathscr{F}_{\text {in }}(\mathscr{H})$, where

$$
K_{s}=K+\bar{K}^{*} \text {. }
$$

Proof: Cf. Ref. 2.

Using Lemma 2.2, we can prove the following lemma.

Lemma 2.3: Let $K, L \in \mathscr{I}_{2}(\mathscr{H})$ and set

$$
\Phi_{v}=\left\langle A^{*}|L| A^{*}\right\rangle^{v} \Omega, \quad v=0,1,2, \ldots
$$

Then

$$
\begin{aligned}
\langle A|K| A\rangle \Phi_{0}= & 0, \quad\langle A|K| A\rangle \Phi_{1}=\operatorname{Tr}\left(K L_{s}\right) \Phi_{0}, \\
\langle A|K| A\rangle \Phi_{v}= & v \operatorname{Tr}\left(K L_{s}\right) \Phi_{v-1}+v(v-1) \\
& \times\left\langle A^{*}\left|L_{s} K \bar{L}_{s}^{*}\right| A^{*}\right\rangle \Phi_{v-2}, \quad v \geqslant 2,
\end{aligned}
$$

where Tr means trace.

Let $\mathbb{C}\left(\mathscr{H}_{1}, \mathscr{H}_{2}\right)$ denote the set of densely defined closed linear operators from a Hilbert space $\mathscr{H}^{2}$, to a Hilbert space $\mathscr{H}_{2}$ and set $\mathbb{C}(\mathscr{H})=\mathbb{C}(\mathscr{H}, \mathscr{H})$.

For $S \in \mathbb{C}(\mathscr{H})$, we denote by $d \Gamma(S)$ the second quantization of $S$. The special case $S=I$ gives the number operator $N$ :

$$
N=d \Gamma(I) .
$$

The following estimates are well known:

$$
\|A(F) \# \Psi\| \leqslant\|F\|\left\|(N+1)^{1 / 2} \Psi\right\|, \quad \Psi \in D\left(N^{1 / 2}\right) .
$$

Lemma 2.4: Let $S \in \mathbb{C}(\mathscr{H})$ and define

$$
\begin{aligned}
\mathscr{D}_{s}= & \mathscr{L}\left\{A\left(F_{1}\right)^{* \cdots} A\left(F_{m}\right) * \Omega,\right. \\
& \left.\Omega \mid F_{j} \in D(S), j=1, \ldots, m, m \geqslant 1\right\} .
\end{aligned}
$$


Let $\left\{e_{n}\right\}_{n}$ be a complete orthonormal system (CONS) of $\mathscr{H}$ with $e_{n} \in D\left(S^{*}\right)$ for all $n$. Then, for all $\Psi \in \mathscr{D}_{S}$,

$$
s-\lim _{N \rightarrow \infty} \sum_{n=1}^{N} A\left(e_{n}\right)^{*} A\left(S^{*} e_{n}\right) \Psi=d \Gamma(S) \Psi .
$$

Proof: An easy exercise.

In the same way as in the proof of Lemma 2.4, we can prove the following fact.

Lemma 2.5: Let $\mathscr{K}$ be a Hilbert space and $S, T \in \mathbb{C}(\mathscr{K}, \mathscr{H})$ such that $D(S) \cap D(T)$ is dense and $S T^{*} \in \mathbb{C}(\mathscr{H})$. Let $\left\{e_{n}\right\}_{n}$ be a CONS of $\mathscr{K}$ with $e_{n} \in D(S) \cap D(T)$ for all $n$. Then, for all $\Phi \in \mathscr{Z}_{S^{*}}$ and $\Psi \in \mathscr{L}_{S T}$,

$s-\lim _{v \rightarrow \infty}\left(\Phi, \sum_{n=1}^{N} A\left(S e_{n}\right)^{*} A\left(T e_{n}\right) \Psi\right)=\left(\Phi, d \Gamma\left(S T^{*}\right) \Psi\right)$.

We here extend a terminology: We say that an operator $T$ is Hilbert-Schmidt if $D(T)$ is dense and the closure is Hilbert-Schmidt. Also in this case we write $T \in \mathscr{T}_{2}(\cdot, \cdot)$.

Lemma 2.6: Let $K \in \mathscr{I}_{2}(\mathscr{H})$ and $S \in \mathbb{C}(\mathscr{H})$ such that $\bar{S}^{*} K_{s}^{*} \in \mathscr{T}_{2}(\mathscr{H})$. Then, for all $v \geqslant 1$, $\left\langle A^{*}|K| A^{*}\right\rangle^{*} \Omega \in D\left(d \Gamma(S)^{*}\right)$ and

$d \Gamma(S)^{*}\left\langle A^{*}|K| A^{*}\right\rangle^{*} \Omega$

$$
=v\left\langle A^{*}\left|K_{s} \bar{S}\right| A^{*}\right\rangle\left\langle A^{*}|K| A^{*}\right\rangle^{v-1} \Omega \text {. }
$$

Proof. Use Lemma 2.4 .

We introduce a subset of $\mathscr{F}_{2}(\mathscr{H})$.

Definition 2.7: We say that $K \in \mathscr{F}_{2}(\mathscr{H})$ is in the set $H S_{1}\left(\mathscr{H}^{\prime}\right)$ if $\|K\|<1$ and $K=\bar{K}^{*}$.

Let

$Z_{x}=\cap_{k=1}^{\infty} D\left(N^{k}\right)$.

Lemma 2.8: Let $K \in H S_{1}(\mathscr{H})$ and $\Psi \in \mathscr{F}_{\text {fin }}(\mathscr{H})$. Then

$$
\begin{gathered}
s-\lim _{n \rightarrow \infty} \sum_{v=0}^{n}(-1)^{v} \frac{\left\langle A^{*}|K| A^{*}\right\rangle^{v} \Psi}{2^{v} v !} \\
\equiv \exp \left\{-\frac{\left\langle A^{*}|K| A^{*}\right\rangle}{2}\right\} \Psi
\end{gathered}
$$

exists and belongs to $\mathscr{D}_{x}$.

Proof: See Refs. 1 and 2.

By virtue of Lemma 2.8, for $K \in H S_{1}(\not{H})$, we can define a vector $\Omega(K) \in \mathscr{Z}_{\infty}$ by

$$
\Omega(K)=N_{0} \exp \left\{-\frac{\left\langle A^{*}|K| A^{*}\right\rangle}{2}\right\} \Omega
$$

Here, $N_{0}>0$ is the normalization constant so that $\|\Omega(K)\|=1$. Explicitly $N_{0}$ is given by

$$
N_{0}=\left\{\operatorname{det}\left(I-K^{*} K\right)\right\}^{1 / 4} \text {, }
$$

where det $(I+A)$ denotes the determinant of $I+A$ with $A$ being trace class (e.g., Refs.1,2, and 11).

Finally we derive an equation satisfied by $\Omega(K)$.

Lemma 2.9: For all $F \in \mathscr{H}$ and $K \in H S_{1}\left(\mathscr{H}^{\prime}\right)$,

$$
\{A(F)+A(K \bar{F}) *\} \Omega(K)=0
$$

Proof: Let

$$
\Omega_{n}(K)=\sum_{v=0}^{n}(-1)^{*} \frac{\left\langle A^{*}|K| A^{*}\right\rangle^{v}}{2^{v} v !} \Omega .
$$

Then, by (2.7) and the fact $K=\bar{K}^{*}$, we have

$$
A(F) \Omega_{n}(K)=-A(K \bar{F}) * \Omega_{n-1}(K) .
$$

Taking the limit $n \rightarrow \infty$ of the both sides, we obtain (2.12).

\section{NONINVERTIBLE BOGOLIUBOV TRANSFORMATIONS}

In this section we consider the case where the Hilbert space $\mathscr{H}$ is given by the direct sum of two Hilbert spaces $\mathscr{K}$ and $\mathscr{K}$ :

$$
\mathscr{H}=\mathscr{K} \oplus \mathscr{M},
$$

so that we have

$$
\mathscr{F}_{s}(\mathscr{H})=\mathscr{F}_{s}(\mathscr{K}) \otimes \mathscr{F}_{s}(\mathscr{M}) .
$$

To avoid notational confusion, we denote by $b(f), f \in \mathscr{K}$ [resp. $a(u), u \in \mathscr{M}$ ] the annihilation operator in $\mathscr{F}_{s}(\mathscr{K})$ [resp. $\left.\mathscr{F}_{s}(\mathscr{M})\right]$. We shall write vectors in $\mathscr{H}$ as $f \oplus u, f \in \mathscr{K}$, $u \in \mathscr{M}$. Under the identification (3.2), we have

$$
\begin{array}{ll}
b(f) \otimes I=A(f \oplus 0), & f \in \mathscr{K}, \\
I \otimes a(u)=A(0 \oplus u), & u \in \mathscr{H} .
\end{array}
$$

Let $V, W \in \mathbb{B}(\mathscr{K})$ and $P, Q \in \mathbb{B}(\mathscr{K}, \mathscr{M})$ satisfying the following conditions:

$$
\begin{aligned}
& \text { (C.1) } W^{*} W-V^{*} V+Q^{*} Q-P^{*} P=I \\
& \text { (C.2) } W^{*} \bar{V}-V^{*} \bar{W}+Q^{*} \bar{P}-P^{*} \bar{Q}=0, \\
& \text { (C.3) } W W^{*}-\bar{V} \bar{V} *=I \\
& \text { (C.4) } V W^{*}-\bar{W} \bar{V}^{*}=0 \\
& \text { (C.5) } Q W^{*}-\bar{P} \bar{V}^{*}=0 \\
& \text { (C.6) } Q V^{*}-\bar{P} \bar{W}^{*}=0 \\
& \text { (C.7) } Q P^{*}-\bar{P} \bar{Q}^{*}=0 \\
& \text { (C.8) the operator } \\
& R=Q Q^{*}-\bar{P} \bar{P} *
\end{aligned}
$$

is bijective.

For each $f \in \mathscr{K}$, we define $B(f)$ by

$$
\begin{aligned}
B(f)= & b(W f) \otimes I+b(\overline{V f})^{*} \otimes I \\
& +I \otimes a(Q f)+I \otimes a(\overline{P f})^{*} .
\end{aligned}
$$

Obviously $\mathscr{F}_{\text {fin }}(\mathscr{H}) \in D(B(f)) \cap D\left(B(f)^{*}\right)$ for all $f \in \mathscr{K}$, which implies that $B(f)^{*}$ is densely defined and hence $B(f)$ is closable. We denote the closure of $B(f)$ by the same symbol. The operator $B(f)^{\#}$ leaves $\mathscr{F}_{\text {fin }}(\mathscr{H})$ invariant. Moreover, conditions (C.1) and (C.2) imply that

$\left[B(f), B(g)^{*}\right]=(f, g), \quad[B(f), B(g)]=0, \quad f, g \in \mathscr{K}^{n}$,

on $\mathscr{F}_{\text {fin }}(\mathscr{H})$, i.e., $B(f)$ \#'s satisfy the CCR. Thus (3.6) gives a Bogoliubov tranformation of $\{b(f) \# \otimes I \mid f \in \mathscr{K}\}$. This Bogoliubov transformation may be noninvertible as the following proposition shows.

Proposition 3.1: Suppose that

$\operatorname{dim} \cap_{f \in x^{\prime}} \operatorname{Ker} B(f)<\infty$.

Then there exist no invertible bounded linear operators $T, U: \mathscr{F}_{s}(\mathscr{H}) \rightarrow \mathscr{F}_{s}(\mathscr{H})$ such that

$$
T(b(f) \otimes I U=B(f), \quad f \in \mathscr{X} .
$$

Proof: Suppose that (3.9) holds with $U$ invertible and let

$$
\begin{aligned}
& \Psi\left(u_{1}, \ldots, u_{n}\right)=U^{-1} I \otimes a\left(u_{1}\right)^{* \cdots} I \otimes a\left(u_{n}\right)^{*} \Omega, \\
& u_{j} \in \mathscr{M}, j=1, \ldots, n, \quad n \geqslant 1 .
\end{aligned}
$$

Since $b(f) \otimes I$ and $I \otimes a(u)$ commute on $\mathscr{F}_{\text {fin }}(\mathscr{K})$ and $(b(f) \otimes I) \Omega=0$, it follows that for all $f \in \mathscr{K}$,

$$
(b(f) \otimes I) U\left(u_{1}, \ldots, u_{n}\right)=0
$$


and hence

$$
B(f) \Psi\left(u_{1}, \ldots, u_{n}\right)=0,
$$

which, however, contradicts (3.8).

In what follows, we shall show that under an additional condition, (3.8) is true and hence the Bogoliubov transformation (3.6) is really noninvertible.

For $A \in \mathbb{B}(\mathscr{K})$ and $B \in \mathbb{B}(\mathscr{K}, \mathscr{M})$, we define $S(A, B) \in \mathbb{B}(\mathscr{K}, \mathscr{H})$ by

$$
S(A, B) f=A f \oplus B f, \quad f \in \mathscr{K} .
$$

For notational simplicity, we set

$$
\bar{S}(A, B)=J S(A, B) J .
$$

It is straightforward to see that $(C .1)-(C .7)$ are equivalent to the following conditions:

$$
\begin{aligned}
& S(W, Q) * S(W, Q)-S(V, P) * S(V, P)=I, \\
& S(W, Q) * \bar{S}(V, P)-S(V, P) * \bar{S}(W, Q)=0, \\
& S(W, Q) S(W, Q)^{*}-\bar{S}(V, P) \bar{S}(V, P)^{*}=S(I, R), \\
& S(V, P) S(W, Q)^{*}-\bar{S}(W, Q) \bar{S}(V, P)^{*}=0 .
\end{aligned}
$$

Lemma 3.2: The operator $S(W, Q)$ is bijective.

Proof: Throughout the proof, we set $S(W, Q)=Y$. We see from (3.12) that

$$
Y^{*} Y \geqslant 1
$$

and that $Y^{*} Y$ is bijective, which implies that $\operatorname{Ran} Y^{*}$ (the range of $Y^{*}$ ) equals $\mathscr{K}^{*}$ and that $Y$ is one to one. It is well known $^{12}$ that for all densely defined closed linear operators $S$ from a Hilbert space to a Hilbert space,

$$
\sigma\left(S^{*} S\right) \backslash\{0\}=\sigma\left(S S^{*}\right) \backslash\{0\} .
$$

Hence (3.16) implies that

$$
\sigma\left(Y Y^{*}\right) \backslash\{0\} \subset[1, \infty) \text {. }
$$

Thus we need only to show that $\operatorname{Ker} Y Y^{*}\left(=\operatorname{Ker} Y^{*}\right)$ $=\{0\}$. Let $F \in \operatorname{Ker} Y^{*}$ so that $Y^{*} F=0$. Then (3.15) and the injectivity of $Y$ give $\bar{S}(V, P)^{*} F=0$. Putting this into (3.14), we get

$$
S(I, R) F=0,
$$

which, together with (C.8), implies that $F=0$.

The proof of Lemma 3.2 shows also that

$$
\sigma(S(W, Q) * S(W, Q))=\sigma\left(S(W, Q) S(W, Q)^{*}\right) \subset[1, \infty)
$$

Lemma 3.2 allows us to define

$$
\begin{aligned}
& X=\bar{S}(V, P) \bar{S}(W, Q)^{-1} \in \mathbb{B}\left(\mathscr{H}^{*}\right) . \\
& \text { Lemma 3.3: The operator } X \text { satisfies } \\
& \bar{X}=X^{*}
\end{aligned}
$$

and

$$
\|X\|<1 .
$$

Proof: Formula (3.19) follows from (3.13) and Lemma

3.2. We have by (3.12)

$$
X^{*} X=I-\left(\bar{Y} \bar{Y}^{*}\right)^{-1} \text {, }
$$

where we put $Y=S(W, Q)$, and hence

$$
\|X F\|^{2}=\|F\|^{2}-\left\|\left(Y Y^{*}\right)^{-1 / 2} \bar{F}\right\|^{2}, \quad F \in \mathscr{H} .
$$

On the other hand, we have

$$
\left\|\left(Y Y^{*}\right)^{-1 / 2} \bar{F}\right\| \geqslant c\|\bar{F}\|=c\|F\|, \quad F \in \mathscr{H} .
$$

with $0<c=\left\|\left(Y Y^{*}\right)^{1 / 2}\right\|^{-1} \leqslant 1[$ cf. (3.17) ]. Therefore,

$$
\|X F\|^{2} \leqslant\left(1-c^{2}\right)\|F\|^{2},
$$

which implies that $\|X\| \leqslant \sqrt{1-c^{2}}<1$. Thus (3.20) follows.

We now state the main result in this section.

Theorem 3.4: Suppose that $X \in \mathscr{F}_{2}$ ( $\left.\mathscr{H}\right)$. Then:

(i) $X$ belongs to $H S_{1}(\mathscr{H})$.

(ii) Let $\Omega(X)$ be given by (2.11) with $K=X$. Then $\Omega(X)$ is a unique (up to constant multiples) vector $\Psi$ in $D(B(f))$ such that for all $f \in \mathscr{K}$,

$$
B(f) \Psi=0,
$$

and the subspace

$$
\begin{aligned}
\mathscr{F}_{\text {fin }}^{B}= & \mathscr{L}\left\{B\left(f_{1}\right)^{*} \cdots B\left(f_{n}\right)^{*} \Omega(X),\right. \\
& \left.\Omega(X) \mid f_{j} \in \mathscr{K}, j=1, \ldots, n, n \geqslant 1\right\}
\end{aligned}
$$

is dense in $\mathscr{F}_{s}(\mathscr{H})$.

Proof: Part (i) follows from the assumption and Lemma 3.3. Note that $B(f)$ can be written as

$$
B(f)=A(S(W, Q) f)+A(\bar{S}(V, P) \bar{f})^{*}
$$

on $D\left(N^{1 / 2}\right)$. Hence, by Lemma 3.2 , for $\Psi \in \mathscr{Z}_{\infty}$, the equations $B(f) \Psi=0, f \in \mathscr{K}$, are equivalent to

$$
\{A(F)+A(X \bar{F}) *\} \Psi=0 .
$$

Hence, (2.12) gives (3.21) with $\Psi=\Omega(X)$.

We next prove the uniqueness of $\Omega(X)$ and that $\mathscr{F}_{\text {fin }}^{B}$ is dense in $\mathscr{F}_{s}(\mathscr{H})$. We denote by $\Omega_{:}$the Fock vacuum in $\mathscr{F}_{s}\left(\mathscr{K}^{r}\right)$. Let $\mathscr{F}^{B}$ be the closure of $\mathscr{F}_{\text {fin }}^{B}$. Define $U: \mathscr{F}_{\text {fin }}^{B} \rightarrow \mathscr{F}_{\text {fin }}(\mathscr{K})$ by

$$
\begin{aligned}
& U \Omega(X)=\Omega_{. h}, \\
& U B\left(f_{1}\right)^{*} \cdots B\left(f_{n}\right) * \Omega(X)=b\left(f_{1}\right)^{*} \cdots b\left(f_{n}\right) * \Omega_{\ldots}, \\
& f_{j} \in \mathscr{K}, j=1, \ldots, n, n \geqslant 1,
\end{aligned}
$$

and extending it by linearity to $\mathscr{F}_{\text {fin }}^{B}$. The operator $U$ is well defined and extends uniquely to a unitary operator from $\mathscr{F} B$ to $\mathscr{F}_{s}(\mathscr{K})$. By Lemma 2.8 and $(2.9)$, we have $\mathscr{F}_{\text {fin }}^{B} \subset \mathscr{D}_{\infty}$. Moreover, using the unitary correspondence between $\mathscr{F}_{\text {fin }}^{\infty}$ and $\mathscr{F}_{\text {in }}(\mathscr{K})$, we can show that every vector in $\mathscr{F}_{\text {fin }}^{B}$ is an analytic vector for the symmetric operator

$$
\Phi_{B}(f)=(1 / \sqrt{2})\left\{B(f)+B(f)^{*}\right\} .
$$

Let $\Phi(F)$ be the Segal field operator in $\mathscr{F}_{s}\left(\mathscr{H}^{\mathcal{H}}\right)$ :

$$
\Phi(F)=(1 / \sqrt{2})\left\{A(F)+A(F)^{*}\right\},
$$

which is essentially self-adjoint on $\mathscr{F}_{\text {fin }}(\mathscr{H})$; we denote the closure by the same symbol. Using (C.3) - (C.7), we have

$$
\begin{aligned}
& b(f) \otimes I=B\left(W^{*} f\right)-B\left(V^{*} \bar{f}\right)^{*}, \quad f \in \mathscr{K}, \quad \\
& I \otimes a(u)=B\left(Q^{*} R-1 u\right)-B\left(P^{*} \bar{R}-1 \bar{u}\right)^{*}, \quad u \in \mathscr{M} .
\end{aligned}
$$

on $D\left(N^{1 / 2}\right)$. Hence, $\Phi(F)$ is written as

$$
\Phi(F)=\Phi_{B}\left(T_{1} F-J T_{2} F\right), \quad F \in \mathscr{H},
$$

where $T_{j} \in \mathbb{B}(\mathscr{H}, \mathscr{K}), j=1,2$, are defined by

$$
\begin{aligned}
& T_{1}(f \oplus u)=W^{*} f+Q^{*} R^{-1} u, \\
& T_{2}(f \oplus u)=\bar{V}^{*} f+\bar{P}^{*} R-{ }^{-1} u .
\end{aligned}
$$


Thus, for all $F \in \mathscr{H}$, every vector in $\mathscr{F}_{\text {in }}^{B}$ is an analytic vector for $\Phi(F)$. It turns out that for all $F \in \mathscr{H}$, exp $i \Phi(F)$ leaves $\mathscr{F}^{B}$ invariant. Since $\left\{\exp i \Phi(F) \mid F \in \mathscr{H}^{p}\right\}$ is irreducible (e.g., Ref. 10, Appendix to X.7, Lemma 1), it follows that $\mathscr{F}^{R}=\mathscr{F}_{s}(\mathscr{H})$, i.e., $\mathscr{F}_{\text {fin }}^{B}$ is dense in $\mathscr{F}_{s}(\mathscr{H})$. Using this result, one can easily prove the uniqueness of $\Omega(X)$.

Theorem 3.4 and Proposition 3.1 imply that, if $X \in \mathscr{I}_{2}(\mathscr{H})$, then the Bogoliubov transformation (3.6) is noninvertible.

In concluding this section, let us represent the operator $X$ explicitly in terms of $\{P, Q, V, W\}$.

Lemma 3.5: For all $f \in \mathscr{K}$ and $u \in \mathscr{M}$,

$S(W, Q)^{-1}(f \oplus u)$

$$
=\left(1+V^{*} V+P^{*} P\right)^{-1}\left(W^{*} f+Q^{*} u\right) .
$$

Proof: Let $T \in \mathbb{B}(\mathscr{H}, \mathscr{K})$ be the operator defined by the right-hand side of (3.26). Since we already know that $S(W, Q)$ is invertible, we need only to show that

$$
T S(W, Q)=I \text {. }
$$

But this easily follows from (C.1).

We can represent $f \oplus u \in \mathscr{H}$ as a column vector:

$$
f \oplus u=\left(\begin{array}{l}
f \\
u
\end{array}\right) \text {. }
$$

In this representation, every $T \in \mathbb{B}(\mathscr{H})$ can be uniquely written as

$$
T=\left(\begin{array}{ll}
T_{11} & T_{12} \\
T_{21} & T_{22}
\end{array}\right),
$$

with $\quad T_{11} \in \mathbb{B}(\mathscr{K}), \quad T_{12} \in \mathbb{B}(\mathscr{M}, \mathscr{K}), \quad T_{21} \in \mathbb{B}(\mathscr{K}, \mathscr{U})$, $T_{22} \in \mathbb{B}(\mathscr{M})$. It is easy to see that $T \in \mathscr{I}_{2}\left(\mathscr{H}^{2}\right)$ if and only if each $T_{i j}$ is Hilbert-Schmidt.

Lemma 3.6: Let $X$ be given by (3.18) and put

$$
Z=\left(1+V^{*} V+P^{*} P\right)^{-1} \text {. }
$$

Then

$$
\bar{X}=\left(\begin{array}{ll}
V Z W^{*} & V Z Q^{*} \\
P Z W^{*} & P Z Q^{*}
\end{array}\right)
$$

Proof: By Lemma 3.5, we have for all $f \in \mathscr{K}$ and $u \in \mathscr{M}$

$$
\begin{aligned}
\bar{X}(f \oplus u) & =S(V, P)\left(Z W^{*} f+Z Q^{*} u\right) \\
& =\left(V S Z^{*} f+V Z Q^{*} u\right) \oplus\left(P Z W^{*} f+P Z Q^{*} u\right),
\end{aligned}
$$

which gives (3.29).

As a corollary of Lemma 3.6, we have the following.

Corollary 3.7: The operator $X$ is Hilbert-Schmidt if and only if $V Z W^{*}, V Z Q^{*}, P Z W^{*}$, and $P Z Q^{*}$ are HilbertSchmidt.

\section{A FAMILY OF SELF-ADJOINT OPERATORS}

The purpose of this section is to show that the (noninvertible) Bogoliubov transformation (3.6) can be used to construct a family of self-adjoint operators $H$, acting in
$\mathscr{F}_{s}(\mathscr{H})$, with the properties described in Introduction. The idea underlying our method is to find $H$ as an operator "diagonalized" by the Bogoliubov transformation.

Let $K \in \mathscr{J}_{2}(\mathscr{M}, \mathscr{K})$, so that it can be expressed as

$$
K=\sum_{n} \lambda_{n}\left(\psi_{n}, \cdot\right) \phi_{n} \quad \text { (the canonical form), }
$$

where $\left\{\psi_{n}\right\}$ (resp. $\left\{\phi_{n}\right\}$ ) is an orthonormal set in $\mathscr{M}$ (resp. $\mathscr{K})$. As in Sec. II, we can define

$$
\begin{aligned}
& \left\langle b^{*}|K| a^{*}\right\rangle=\sum_{n} \lambda_{n} b\left(\phi_{n}\right)^{*} \otimes I \cdot I \otimes a\left(\bar{\psi}_{n}\right)^{*}, \\
& \langle b|K| a\rangle=\sum_{n} \lambda_{n} b\left(\bar{\phi}_{n}\right) \otimes I \cdot I \otimes a\left(\psi_{n}\right) .
\end{aligned}
$$

on $\mathscr{F}_{\text {in }}(\mathscr{H})$. Similarly, for $M \in \mathscr{F}_{2}(\mathscr{K}, \mathscr{M})$, we can define the operator $\left\langle a^{\#}|M| b^{\#}\right\rangle$ on $\mathscr{F}_{\text {in }}(\mathscr{H})$. We have

$$
\langle b|K| a\rangle^{*}=\left\langle a^{*}\left|K^{*}\right| b^{*}\right\rangle
$$

on $\mathscr{F}_{\text {fin }}(\mathscr{H})$. The following fact is easily proved.

Lemma 4.1: Let $K \in \mathscr{F}_{2}(\mathscr{H})$ and $K=\left\{K_{i j}\right\}$ be the matrix representation of $K$ as in (3.27). Then

$$
\begin{aligned}
\left\langle A^{\#}\right| & K\left|A^{\#}\right\rangle \\
= & \left\langle b^{\#}\left|K_{11}\right| b^{\#}\right\rangle \otimes I+\left\langle b^{\#}\left|K_{12}\right| a^{\#}\right\rangle \\
& +\left\langle a^{\#}\left|K_{21}\right| b^{\#}\right\rangle+I \otimes\left\langle a^{\#}\left|K_{22}\right| a^{\#}\right\rangle
\end{aligned}
$$

on $\mathscr{F}_{\text {fin }}(\mathscr{H})$.

For a densely defined closable linear operator $L: \mathscr{M} \rightarrow \mathscr{K}$ and $L^{\prime}: \mathscr{K} \rightarrow \mathscr{M}$ (we denote their closure by the same symbol, respectively), we define $\left\langle b^{*}|L| a\right\rangle$ and $\left\langle a^{*}\left|L^{\prime}\right| b\right\rangle$ by

$$
\begin{aligned}
& \left\langle b^{*}|L| a\right\rangle=d \Gamma\left(\begin{array}{ll}
0 & L \\
0 & 0
\end{array}\right), \\
& \left\langle a^{*}\left|L^{\prime}\right| b\right\rangle=d \Gamma\left(\begin{array}{ll}
0 & 0 \\
L^{\prime} & 0
\end{array}\right) .
\end{aligned}
$$

In what follows, we assume also

$$
\text { (C.9) } X \in \mathscr{I}_{2}(\mathscr{H}) \text {. }
$$

Let $h$ be a non-negative self-adjoint operator in $\mathscr{K}$ such that

Ker $h=\{0\}, J h J=h$, and the following (C.10)-(C.12) are satisfied.

(C.10) The following operators are all HilbertSchmidt:

$V h W^{*}, V h Q^{*}, P h W^{*}, P h Q^{*}, W h V^{*}, Q h V^{*}, W h P^{*}, Q h P^{*}$ $h^{1 / 2} V^{*}, h^{1 / 2} P^{*}$.

(C.11) The operators $W h W^{*}+\bar{V} h \bar{V}^{*}$ and $Q h Q^{*}+\bar{P} h \bar{P}^{*}$ are self-adjoint.

(C.12) The operators $W h Q^{*}+\bar{V} h \bar{P}^{*}$ and $Q h W^{*}+\bar{P} h \bar{V}^{*}$ are densely defined and closable.

Conditions (C.10)-(C.12) allow us to define

$$
\begin{aligned}
H= & d \Gamma\left(W h W^{*}+\bar{V} h \bar{V}^{*}\right) \otimes I+I \otimes d \Gamma\left(Q h Q^{*}+\bar{P} h \bar{P}^{*}\right)+\left\langle b^{*}\left|W h Q^{*}+\bar{V} h \bar{P}^{*}\right| a\right\rangle+\left\langle a^{*}\left|Q h W^{*}+\bar{P} h \bar{V}\right| b\right\rangle \\
& +\left\langle b^{*}\left|W h V^{*}\right| b^{*}\right\rangle \otimes I+I \otimes\left\langle a^{*}\left|Q h P^{*}\right| a^{*}\right\rangle+\left\langle b^{*}\left|Q h V^{*}\right| a^{*}\right\rangle+\left\langle a^{*}\left|W h P^{*}\right| b^{*}\right\rangle+\left\langle b\left|V h W^{*}\right| b\right\rangle \otimes I \\
& +I \otimes\left\langle a\left|P h Q^{*}\right| a\right\rangle+\left\langle b\left|P h W^{*}\right| a\right\rangle+\left\langle a\left|V h Q^{*}\right| b\right\rangle
\end{aligned}
$$


Condition (C.12) implies that $D\left(h \vec{V}^{*}\right) \cap D\left(h W^{*}\right)$ and $D\left(h \bar{P}^{*}\right) \cap D\left(h Q^{*}\right)$ are dense in $\mathscr{K}$ and in $\mathscr{H}$, respectively. Hence, the subspace

$$
\begin{aligned}
D_{0}(H)= & \mathscr{L}\left\{A\left(f_{1} \oplus u_{1}\right)^{* \cdots} A\left(f_{n} \oplus u_{n}\right)^{*} \Omega \mid f_{j}\right. \\
& \left.\in D\left(h \bar{V}^{*}\right) \cap D\left(h W^{*}\right), u_{j} \in D\left(h \bar{P}^{*}\right) \cap D\left(h Q^{*}\right)\right\}
\end{aligned}
$$

is dense in $\mathscr{F}_{s}(\mathscr{H})$. It is easy to see that $D_{0}(H) \subset D(H)$. The main result in this section is the following theorem.

Theorem 4.2: The operator $H$ is essentially self-adjoint on $D_{0}(H)$ and

$$
H \geqslant E \text {, }
$$

with

$$
E=-\left\|V h^{1 / 2}\right\|_{\mathrm{HS}}^{2}-\left\|P h^{1 / 2}\right\|_{\mathrm{ISS}}^{2},
$$

where $\|\cdot\|_{\text {HS }}$ denotes Hilbert-Schmidt norm. Moreover, the closure of the operator

$$
\widehat{H} \equiv H-E
$$

is unitarily equivalent to $d \Gamma(h)$ acting in $\mathscr{F}_{s}(\mathscr{K})$.

To prove this theorem, we prepare somc lemmas. The following lemma explains the origin of $H$.

Lemma 4.3: Let $\left\{e_{n}\right\}_{n} \subset D\left(h^{1 / 2}\right)$ be a CONS of $\mathscr{K}^{\gamma}$ and define

$$
H_{\Lambda}=\sum_{n=1}^{\vee} B\left(h^{1 / 2} e_{n}\right) * B\left(h^{1 / 2} e_{n}\right) .
$$

Then, for all $\Psi, \Phi \in D_{0}(H)$,

$$
\lim _{\hat{V} \rightarrow \infty}\left(\Phi, H_{N} \Psi\right)=(\Phi, \widehat{H} \Psi) \text {. }
$$

Proof: We set $T=S(V, P)$ and $Y=S(W, Q)$. Using (3.23) and (2.3), we have

$$
\left(\Phi, H_{N} \Psi\right)=S_{N}^{(1)}+S_{N}^{(2)}+S_{N}^{(3)},
$$

where

$$
\begin{aligned}
S_{N}^{(1)}= & \left(\Phi, \sum_{n=1}^{N}\left\{A\left(Y h^{1 / 2} e_{n}\right)^{*} A\left(Y h^{1 / 2} e_{n}\right)\right.\right. \\
& \left.\left.+A\left(\bar{T} h^{1 / 2} \bar{e}_{n}\right)^{*} A\left(\bar{T} h^{1 / 2} \bar{e}_{n}\right)\right\} \Psi\right), \\
S_{N}^{(2)}= & \left(\Phi, \sum_{n=1}^{N}\left\{A\left(Y h^{1 / 2} e_{n}\right)^{*} A\left(\bar{T} h^{1 / 2} \bar{e}_{n}\right)^{*}\right.\right. \\
& \left.\left.+A\left(Y h^{1 / 2} e_{n}\right) A\left(\bar{T} h^{1 / 2} \bar{e}_{n}\right)\right\} \Psi\right), \\
S_{N}^{(3)}= & \sum_{n=1}\left\|\bar{T} h^{1 / 2} e_{n}\right\|^{2} .
\end{aligned}
$$

By Lemma 2.5, we see that

$$
\lim _{v \rightarrow \infty} S_{N}^{(1)}=\left(\Phi,\left\{d \Gamma\left(Y h Y^{*}\right)+d \Gamma\left(\bar{T} h \bar{T}^{*}\right)\right\} \Psi\right)
$$

Moreover, we have

$\lim _{l \rightarrow \infty} S_{. i}^{(2)}=\left(\Phi\left(\left\langle A^{*}\left|Y h T^{*}\right| A^{*}\right\rangle+\left\langle A\left|T^{\prime} h Y^{*}\right| A\right\rangle\right) \Psi\right)$,

$\lim _{x \rightarrow \infty} S_{i}^{(3)}=-E$
On the other hand, it is straightforward to see that

$$
\left\{d \Gamma\left(Y h Y^{*}\right)+d \Gamma\left(\bar{T} h \bar{T}^{*}\right)+\left\langle A^{*}\left|\bar{T} h \bar{Y}^{*}\right| A^{*}\right\rangle\right.
$$$$
\left.+\left\langle A\left|\bar{Y} h \bar{T}^{*}\right| A\right\rangle\right\} \Psi=H \Psi \text {. }
$$

Thus (4.13) follows.

Lemma 4.4: The operator $\widehat{H}$ is symmetric and non-negative. Moreover, the commutation relations

$$
[\hat{H}, B(f)]=-B(h f), \quad f \in D(h),
$$

hold on $D_{0}(H)$.

Proof: The non-negativity (hence symmetricity) of $\hat{H}$ follows from that of $H_{N}$ and Lemma 4.3. We have for all $\Psi, \Phi \in D_{0}(H)$

$$
\begin{aligned}
& \left(\Phi,\left[H_{N}, B(f)\right] \Psi\right) \\
& \quad=-\left(\Phi, \sum_{n=1}^{N}\left(f, h^{1 / 2} e_{n}\right) B\left(h^{1 / 2} e_{n}\right) \Psi\right) \\
& \quad \rightarrow-(\Phi, B(h f) \Psi)
\end{aligned}
$$

as $N \rightarrow \infty$, which, together with Lemma 4.3, implies (4.15).

Lemma 4.5: Let $\Omega(X)$ be as in Theorem 3.4. Then, $\Omega(X) \in D(H)$ and

$$
\hat{H} \Omega(X)=0 \text {. }
$$

Proof: Set $T=S(V, P), Y=S(W, Q)$. Using Lemmas 2.3, 2.6, and (4.14), we have

$$
\begin{aligned}
\hat{H} \Omega(X)= & \left\{\left\langle A^{*}\left|\bar{T} h \bar{Y}^{*}\right| Y^{*}\right\rangle+\left\langle A^{*}\left|X \bar{Y} h \bar{T}^{*} X\right| A^{*}\right\rangle\right. \\
& -\left\langle A^{*}\left|X \bar{Y} h \bar{Y}^{*}\right| A^{*}\right\rangle-\left\langle A^{*}\left|X T h T^{*}\right| A^{*}\right\rangle \\
& \left.-\operatorname{Tr}\left(\bar{Y}^{*} h \bar{T}^{*} X\right)+\left\|T h^{1 / 2}\right\|_{\mathrm{HS}}^{2}\right\} \Omega(X) .
\end{aligned}
$$

Note that

$$
\begin{aligned}
& \operatorname{Tr}\left(\bar{T} h \bar{Y}^{*} X\right)=\operatorname{Tr}\left(\bar{T} h \bar{T}^{*}\right)=\left\|T h^{1 / 2}\right\|_{\mathrm{HS}}^{2}, \\
& X \bar{Y} h \bar{Y}^{*}=\bar{T} h \bar{Y} *, \quad X \bar{Y} h \bar{T}{ }^{*} X=\bar{T} h \bar{T}{ }^{*} X,
\end{aligned}
$$

and

$$
\left\langle A^{*}\left|\bar{T} h \bar{T}^{*} X\right| A^{*}\right\rangle=\left\langle A^{*}\left|X T h T^{*}\right| A^{*}\right\rangle,
$$

since $\bar{X}=X^{*}$. Thus (4.16) follows.

Proof of Theorem 4.2: We have already proved (4.9) (Lemma 4.4). Let $\mathscr{F}_{\mathrm{fin}}^{B}, \mathscr{F}^{B}$, and $U$ be as in the proof of Theorem 3.4. Define

$$
L=\widehat{H} \uparrow D_{0}(H) .
$$

Let $\Psi \in D_{0}(H)$ and $f_{j} \in D(h), j=1, \ldots, n$. Then, by $(4.16)$ and (4.15), we have

$$
\begin{aligned}
0= & \left(\hat{H} \Omega(X), B\left(f_{1}\right) \cdots B\left(f_{n}\right) \Psi\right) \\
= & -\left(\Omega(X), \sum_{j=1}^{n} B\left(f_{1}\right) \cdots B\left(h f_{j}\right) \cdots B\left(f_{n}\right) \Psi\right) \\
& +\left(\Omega(X), B\left(f_{1}\right) \cdots B\left(f_{n}\right) L \Psi\right),
\end{aligned}
$$

which implies that $B\left(f_{1}\right) * \cdots B\left(f_{n}\right) * \Omega(X) \in D\left(L^{*}\right)$ and

$$
\begin{aligned}
L^{*} B & \left(f_{1}\right)^{*} \cdots B\left(f_{n}\right) * \Omega(X) \\
\quad= & \sum_{j=1}^{n} B\left(f_{1}\right) * \cdots B\left(h f_{j}\right) * \cdots B\left(f_{n}\right) * \Omega(X) .
\end{aligned}
$$

Hence, we obtain

$$
U L^{*} U^{-1}=d \Gamma(h)
$$


on the subspace

$$
\begin{aligned}
D_{0}(d \Gamma(h))= & \mathscr{L}\left\{b\left(f_{3}\right)^{*} \cdots b\left(f_{n}\right)^{*} \Omega_{. \not{ }},\right. \\
& \left.\Omega_{\mathscr{H}} \mid f_{j} \in D(h), j=1, \ldots, n, n \geqslant 1\right\} .
\end{aligned}
$$

Since $D_{0}(d \Gamma(h))$ is a core of $d \Gamma(h)$ and $L^{*}$ is closed, it follows that $L^{*}$ is self-adjoint. By a general theorem, $L^{* *}$ equals $\bar{L}$, the closure of $L$. Thus $\bar{L}$ is self-adjoint, i.e., $L$ is essentially self-adjoint. The unitary equivalence of $\bar{L}$ to $d \Gamma(h)$ follows from (4.17).

It is obvious that $H$ can be rewritten as $H=H_{0}+H_{I}$ with $H_{0}$ given by (1.1). Thus we have accomplished the main purpose of the present paper.

\section{SCATTERING THEORY}

In this section we discuss scattering theory associated with the pair $\left(H_{0}, H\right)$, where $H_{0}$ is given by (1.1).

Lemma 5. 1: For all $f \in D\left(h^{-1 / 2}\right)$ and $\Psi \in D\left(\hat{H}^{1 / 2}\right)$,

$\|B(f) \Psi\| \leqslant\left\|h^{-1 / 2} f\right\|\left\|\hat{H}^{1 / 2} \Psi\right\|$,

$\left\|B(f)^{*} \Psi\right\| \leqslant\left\|h^{-1 / 2} f\right\|\left\|\hat{H}^{1 / 2} \Psi\right\|+\|f\|\|\Psi\|$.

Proof: Since $B(f)^{*} B(f) \geqslant 0, H_{N}$ is monotone increasing in $N$. Hence, it follows from (4.13) that for all $n$,

$$
\left\|B\left(h^{1 / 2} e_{n}\right) \Psi\right\|^{2} \leqslant\left\|\hat{H}^{1 / 2} \Psi\right\|^{2}, \quad \Psi \in D_{0}(H) .
$$

Taking $e_{n}=h^{-1 / 2} f /\left\|h^{-1 / 2} f\right\|\left(f \in D\left(h^{-1 / 2}\right)\right)$, we obtain (5.1) with $\Psi \in D_{0}(H)$. Since $D_{0}(H)$ is also a core of $\hat{H}^{1 / 2}$, this result extends to all $\Psi \in D\left(\hat{H}^{1 / 2}\right)$. Inequality (5.2) follows from (3.7), (5.1), and a limiting argument.

Lemma 5.2: For all $f \in D\left(h^{-1 / 2}\right) \cap D(h)$ and $\Psi, \Phi \in D(H)$,

$$
(H \Phi, B(f) \Psi)-\left(B(f)^{*} \Phi, H \Psi\right)--(\Phi, B(h f) \Psi) .
$$

Proof: For all $\Psi, \Phi \in D_{0}(H),(5.3)$ follows from (4.15). Using (5.1) and the fact that $D_{0}(H)$ is a core of $H,(5.3)$ with $\Psi, \Phi \in D_{0}(H)$ extends to all $\Psi, \Phi \in D(H)$.

Lemma 5.3: For all $f \in D\left(h^{-1 / 2}\right)$ and $\Psi \in D\left(\hat{H}^{1 / 2}\right)$,

$e^{i t H} B(f) e^{-i t H} \Psi=B\left(e^{i t h} f\right) \Psi$.

Proof: Let $\{e(\lambda)\}$ be the spectral family of $h$ and

$\mathscr{E}=\bigcup_{n=1}^{\infty} \operatorname{Ran} e[1 / n, n]$.

For $\Psi, \Phi \in D(H)$ and $f \in \mathscr{E}$, we define

$$
F(t)=\left(e^{-i t H} \Phi, B(f) e^{-i t H} \Psi\right) .
$$

Then, using Lemma 5.2, we can show that $F$ is infinitely many times differentiable in $t$ and

$$
\frac{d^{n} F(t)}{d t^{n}}=\left(e^{-i t H} \Phi, B\left((i h)^{n} f\right) e^{-i t H} \Psi\right), \quad n \geqslant 1 .
$$

Noting that

$$
\sum_{n=0}^{\infty} \frac{\left\|h^{n-1 / 2} f\right\|}{n !}|t|^{n}<\infty
$$

and using (5.1), we see that

$$
\begin{aligned}
F(t) & =\lim _{N \rightarrow \infty} \sum_{n=0}^{N} \frac{\left(\Phi, B\left((\text { ih })^{n} f\right) \Psi\right)}{n !} t^{n} \\
& =\left(\Phi, B\left(e^{i t h} f\right) \Psi\right) .
\end{aligned}
$$

Thus (5.4) with $\Psi \in D(H)$ and $f \in \mathscr{C}$ follows. Once this is proved, a limiting argument allows us to obtain the desired result.

In what follows, in addition to (C.1)-(C.12), we assume the following two conditions.

(C.13) For $\lambda=0,-1 / 2, h^{\lambda} V^{*}$ is compact.

(C.14) There exists an operator $\left[h, W^{*}\right]_{w}$ defined on a dense domain $D_{0}(h) \subset D(h)$ such that for all $f g \in D_{0}(h)$,

$$
\left(h f, W^{*} g\right)-(W f, h g)=\left(f,\left[h, W^{*}\right]_{w} g\right)
$$

and

$$
\int_{-\infty}^{\infty}\left\|h^{\lambda}\left[h, W^{*}\right]_{\omega} e^{-i t h} f\right\| d t<\infty, \quad \lambda=0,-\frac{1}{2} .
$$

We introduce

$$
\begin{aligned}
\mathscr{K}_{0}= & \left\{f \in P_{\mathrm{ac}}(h) \cap D_{0}(h) \cap D\left(h^{-1 / 2}\right) \mid W^{*} e^{i t h} f,\right. \\
& \left.V^{*} e^{i t h} f \in D\left(h^{-1 / 2}\right) \text { for all } t \in \mathbb{R}\right\},
\end{aligned}
$$

where $P_{\mathrm{ac}}(h)$ is the subspace of absolute continuity with respect to $h$.

For $t \in \mathbb{R}$ and $f \in \mathscr{K}_{0}$, we define

$$
b_{t}(f)=e^{i t H} b\left(e^{-i t h} f\right) \otimes I e^{-i t H} .
$$

By virtue of Lemmas $5.1,5.3$, and $(3.24), b_{t}(f)$ is well defined on $D\left(\widehat{H}^{1 / 2}\right)$ and

$$
b_{t}(f)=B\left(e^{i t h} W^{*} e^{-i t h f}\right)-B\left(e^{i t h} V^{*} e^{i l i \bar{f}}\right)^{*}
$$

on $D\left(\hat{H}^{1 / 2}\right)$. We want to show that $b_{t}(f)$ converges as $t \rightarrow \pm \infty$. By (C.14), we can define

$T_{ \pm} f=W^{*} f+i \int_{0}^{ \pm \infty} e^{i t h}\left[h, W^{*}\right]_{w} e^{-i t h} f d t, \quad f \in D_{0}(h)$,

where the integral is taken as a $\mathscr{K}$-valued strong integral.

Theorem 5.4: For all $\Psi \in D\left(\hat{H}^{1 / 2}\right)$ and $f \in \mathscr{K}^{\prime}{ }_{0}$, the strong limits

$$
s-\lim _{t \rightarrow \pm x} b_{t}\left(f^{\prime}\right) \Psi \equiv b_{ \pm}(f) \Psi
$$

exist and are explicitly given by

$$
b_{ \pm}(f) \Psi=B\left(T_{ \pm} f\right) \Psi .
$$

Proof: Let $f \in \mathscr{K}_{0}$ and $\Psi \in D\left(\hat{H}^{\mathrm{t} / 2}\right)$. By (C.13) and an application of Lemma 2 in Ref. 13 (p. 24), we have

$$
s-\lim _{|t| \rightarrow \infty} h^{\lambda} e^{i t h} V^{*} e^{i t h} f=0, \quad \lambda=0,-\frac{1}{2},
$$

which, combined with (5.2), imply that

$$
s-\lim _{\mid t ! \rightarrow \infty} B\left(e^{i t h} V * e^{i t h \bar{f}}\right) * \Psi=0 .
$$

Let

$$
T_{t} f=e^{i t h} W^{*} e^{-i t h} f
$$

Then, differentiating the function $\left(g, T_{f} f\right)\left(g \in D_{0}(h)\right)$ in $t$ first and then integrating the derivative from 0 to $t$, we obtain

$$
T_{t} f=W^{*} f+i \int_{0}^{t} e^{i s h}\left[h, W^{*}\right]_{w} e^{-i s h} f d s .
$$

By (C.14), we see that

$$
s-\lim _{l \rightarrow \pm \infty} h^{-1 / 2} T, f=h^{-1 / 2} T_{ \pm} f
$$


and hence, by $(5.1)$,

$$
s-\lim _{t \rightarrow \pm \infty} B\left(T_{t} f\right) \Psi=B\left(T_{ \pm} f\right) \Psi,
$$

which, together with (5.7) and (5.11), give (5.9) and (5.10).

Physically the operators $b_{ \pm}(f)$ correspond to the annihilation operators of the asymptotic free fields in regard to the degrees of freedom associated with the Hilbert space $F_{s}(\mathscr{K})$.

$$
\begin{aligned}
\text { Let } & \mathscr{F}_{ \pm}= \\
& \mathscr{L}\left\{b_{ \pm}\left(f_{1}\right)^{*} \cdots b_{ \pm}\left(f_{n}\right) * \Omega(X),\right. \\
& \left.\Omega(X) \mid f_{j} \in \mathscr{K}_{0}, j=1, \ldots, n, n \geqslant 1\right\} .
\end{aligned}
$$

We define the " $S$-matrix" $S: \mathscr{F}_{-} \rightarrow \mathscr{F}$, by

$$
\begin{aligned}
S b_{--} & \left(f_{1}\right)^{* \cdots} b_{-}\left(f_{n}\right)^{*} \Omega(X) \\
& =b_{+}\left(f_{1}\right)^{* \cdots} b_{+}\left(f_{n}\right)^{*} \Omega(X) .
\end{aligned}
$$

The $S$-matrix elements are defined by

$$
\begin{aligned}
& S_{n m}\left(f_{1}, \ldots, f_{n} ; g_{1}, \ldots, g_{m}\right) \\
& =\left(b_{-}\left(f_{1}\right)^{* \cdots} b_{-}\left(f_{n}\right) * \Omega(X),\right. \\
& \left.S b_{-}\left(g_{1}\right)^{* \cdots b_{-}}\left(g_{m}\right) * \Omega(X)\right) \text {. }
\end{aligned}
$$

One easily sees that

$$
S_{n m}=\delta_{n m} S_{n n}
$$

and $S_{n n}$ can be written as a sum of $n$ products of $S_{11}$ which is given by

$$
S_{11}(f, g)=\left(f, T_{-}^{*} T_{+} g\right) .
$$

\section{EXAMPLES}

In this section we briefly mention some QFT examples to which the abstract theory in the preceding sections is applicable.

\section{A. Models of a one-dimensional quantum harmonic oscillator coupled to a quantized scalar field}

Let

$$
\mathscr{R}^{r}=L^{2}\left(\mathbb{R}^{d}\right), \quad \mathscr{M}=\mathbb{C},
$$

so that

$$
\mathscr{H}^{\prime}=L^{2}\left(\mathbb{R}^{d}\right) \oplus \mathbb{C}
$$

and

$$
\mathscr{F}_{s}(\mathscr{K})=\mathscr{F}_{s}\left(L^{2}\left(\mathbb{R}^{d}\right)\right) \otimes L^{2}(\mathbb{R}),
$$

In this framework we can construct explicitly two classes of operators $\{P, Q, V, W, h\}$ satisfying (C.1)-(C.14) and show that the corresponding operator $H$ provides the Hamiltonians of various models of a one-dimensional quantum harmonic oscillator coupled to a quantized scalar field over the $d$-dimensional space $\mathbb{R}^{d}$ (e.g., Refs. 4, 5, 7, 8, and 14). See Ref. 8 for the details.

\section{B. Models of a harmonically bound electron interacting with a quantized radiation field}

These models have been discussed from various points of view (e.g., Refs. 6, 15-21). The mathematical framework for these models is given by the following choice of $\{\mathscr{K}, \mathscr{M}\}$ :

$$
\begin{aligned}
& \mathscr{K}=\underbrace{L^{2}\left(\mathbb{R}^{d}\right) \oplus \cdots \oplus L^{2}\left(\mathbb{R}^{d}\right)}_{d-1 \text { times }}, \\
& \mathscr{H}=\mathbb{C}^{d},
\end{aligned}
$$

where we assume that the electron moves in $\mathbb{R}^{d}$ and the radiation field is over $\mathbb{R}^{d}(d \geqslant 2)$. The Coulomb gauge is used for the radiation field. In Ref. 21, the present author discussed one of such models whose Hamiltonian is given by

$$
L=\left(1 / 2 m_{0}\right)(\mathbf{p}-e \mathbf{A}(\rho))^{2}+H_{F}+\frac{1}{2} \epsilon \mathbf{q}^{2},
$$

where $m_{0}>0$ (resp. $\epsilon>0, e \in \mathbb{R} \backslash\{0\}$ ) is a parameter denoting the bare mass of the electron (resp. the spring constant, the elementary charge $), \quad \mathbf{q}=\left(q_{1}, \ldots, q_{d}\right) \in \mathbb{R}^{d}, \mathbf{p}$ $=\left(-i \partial / \partial q_{1}, \ldots,-i \partial / \partial q_{d}\right), \mathbf{A}(\rho)$ is the time-zero radiation field smeared with a suitable function $\rho$ on $\mathbb{R}^{d}$, and $H_{F}$ is the free Hamiltonian of the radiation field. By an explicit construction, we can show that there exists a quintuple $\{P, Q, V, W, h\}$ giving this model. This can be done using results in Ref. 21.

We can also consider another Hamiltonian

$$
L^{\prime}=\frac{1}{2 m_{0}} \mathbf{p}^{2}+\frac{1}{2} \epsilon \mathbf{q}^{2}+H_{F}-\frac{e}{m} \mathbf{p} \cdot \mathbf{A}(\rho),
$$

where $m$ is a renormalized mass of the electron defined by

$$
\frac{1}{m_{0}}=\frac{1}{m}+\left(\frac{e}{m}\right)^{2}\left(\frac{d-1}{d}\right) \int d \mathbf{k} \frac{\hat{\rho}(\mathbf{k})^{2}}{\omega(\mathbf{k})^{2}}
$$

[ $\hat{\rho}$ : the Fourier transform of $\rho, \omega(\mathbf{k})$ : one free photon energy with momentum $\mathbf{k}$ ] This Hamiltonian is obtained by dropping $\mathbf{A}^{2}$ term in the Hamiltonian $L$ given above and renormalizing the electron mass in the way just indicated (cf. Refs. $16,17,22$, and 23 ). The mass renormalization makes $L^{\prime}$ nonnegative. This model is also described in the framework in Secs. III and IV.

\section{ACKNOWLEDGMENT}

This work was supported by the Grant-in-Aid 02740094 for science research from the Ministry of Education, Japan.

${ }^{1}$ E. A. Berezin, The Method of Second Quantization (Academic, New York, 1966).

${ }^{2}$ S. N. M. Ruijsenaars, J. Math. Phys. 18, 517 (1977).

${ }^{3}$ S. N. M. Ruijsenaars, Ann. Phys. (NY) 116, 105 (1978).

${ }^{4}$ A. Arai, J. Math. Phys. 22, 2539 (1981).

${ }^{5}$ A. Arai, J. Math. Phys. 22, 2549 (1981).

${ }^{6}$ A. Arai, J. Math. Phys. 24, 1896 (1983).

${ }^{7}$ A. Arai, J. Math. Anal. Appl. 140, 270 (1989).

${ }^{8}$ A. Arai, Hokkaido Math. J. 19, 1 (1990).

${ }^{9}$ M. Reed and B. Simon, Methods of Modern Mathematical Physics I: Functional Analysis (Academic, New York, 1972).

${ }^{10} \mathrm{M}$. Reed and B. Simon, Methods of Modern Mathematical Physics II: Fourier Analysis, Self-adjointness (Academic, New York, 1975).

${ }^{11}$ M. Reed and B. Simon, Methods of Madern Mathematical Physics IV: Analysis of Operators (Academic, New York, 1978).

${ }^{12}$ P. A. Deift, Duke Math. J. 45, 267 (1978).

${ }^{13} \mathrm{M}$. Reed and B. Simon, Methods of Modern Mathematical Physics III: Scattering Theory (Academic, New York, 1979).

${ }^{14}$ F. Schwabl and W. Thirring, Ergeb. Exakt. Naturwiss. 36, 219 (1964).

${ }^{15}$ N. G. van Kampen, Det Kongelige Danske Videnskabernes Selskab Mat.-Fys. Medd. 26, No. 15 (1951). 
${ }^{16}$ D. P. L. Castrigiano and N. Kokiantonis, Phys. Rev. A 35, 4122 (1987). ${ }^{17}$ D. P. L. Castrigiano and N. Kokiantonis, J. Phys. A: Math. Gen. 20, 4237 (1987).

${ }^{18}$ W. Eckhardt, Z. Phys. B-Condensed Matter 64, 515 (1986).

${ }^{19}$ G. W. Ford, J. T. Lewis, and R. F. O'Connel, Phys. Rev. Lett. 55, 2273 (1985).
${ }^{20}$ G. W. Ford, J. T. Lewis, and R. F. O'Connel, Ann. Phys. (NY) 185, 270 (1988).

${ }^{21}$ A. A rai, "Long-time behavior of an electron interacting with a quantized radiation field," J. Math. Phys. (in press).

${ }^{22}$ P. Blanchard, Commun. Math. Phys. 15, 156 (1969).

${ }^{23}$ A. Arai, J. Math. Phys. 31, 2653 (1990). 
Journal of Mathematical Physics is copyrighted by the American Institute of Physics (AIP). Redistribution of journal material is subject to the AIP online journal license and/or AIP copyright. For more information, see http://ojps.aip.org/jmp/jmpcr.jsp Copyright of Journal of Mathematical Physics is the property of American Institute of Physics and its content may not be copied or emailed to multiple sites or posted to a listserv without the copyright holder's express written permission. However, users may print, download, or email articles for individual use. 